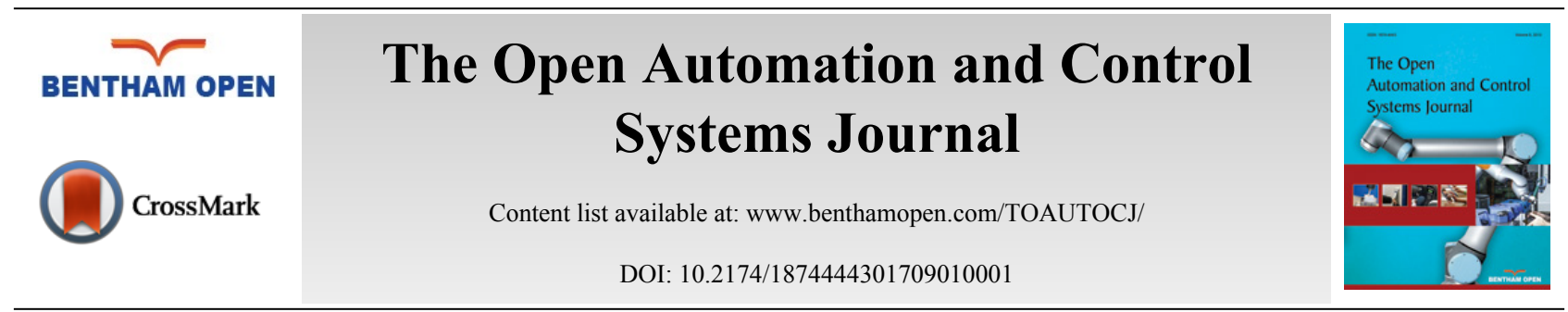

EDITORIAL

\title{
Production and Logistics Systems Optimization (PLSO)
}

The special issue "Production and Logistics Systems Optimization (PLSO)", devoted to the $8^{\text {th }}$ international conference «Integrated Design and Production» (CPI'2013) is composed of five papers. The first paper entitled "Adaptation of memetic algorithm with population management for the improvement of the performances of flexible manufacturing systems" and the second entitled "Investigations on genetic algorithm performances in a parallel machines scheduling environment" are focused on the adaptation of an optimization method based on metaheuristic. The authors propose new methods to improve the results obtained in the literature. The simulation results confirm the effectiveness of the proposed approaches. In the third paper entitled, "Monitoring of Dynamical Systems using Hybrid Automata with Stopwatch. The authors propose a monitoring approach for interruption in hybrid dynamical systems, the principle of which is based on system modeling using hybrid automaton with stopwatch. The results obtained show that the monitoring system is capable to rapidly detect the considered types of faults. The authors develop in the paper four entitled, Optimization as an embodiment design tool to improve mechatronic devices: a novel design approach", a novel mechatronic system design approach in order to consider optimization as a tool that can be used within the embodiment design process to build mechatronic solutions from a set of solution concepts designed with innovative or routine design methods. The aim of paper 5 entitled, "Maintenance Optimizing of Production Systems by Reliability: Different Methods Applied" is to diagnose failure equipment that present daily production stops and are expensive for maintenance and for the company. The authors develop tools such as Pareto and AMDEC model for optimizing maintenance of the workshop boiler at the manufacturing state "Denitex Tlemcen".

Faycal Belkaid (Guest Editor) Manufacturing Engineering Laboratory of Tlemcen University of Tlemcen Tlemcen, Algeria E-mail: f_belkaid@yahoo.fr

Zaki Sari (Guest Editor) Manufacturing Engineering Laboratory of Tlemcen University of Tlemcen Tlemcen, Algeria

Mohammed Dahane (Guest Editor) LGIPM Research Laboratory National School of Engineering of Metz (ENIM) Metz, France

(C) 2017 Belkaid et al.

This is an open access article distributed under the terms of the Creative Commons Attribution 4.0 International Public License (CC-BY 4.0), a copy of which is available at: https://creativecommons.org/licenses/by/4.0/legalcode. This license permits unrestricted use, distribution, and reproduction in any medium, provided the original author and source are credited. 